\title{
A class of wavelet-based Rayleigh-Euler beam element for analyzing rotating shafts
}

\author{
Jiawei Xiang ${ }^{\mathrm{a}, \mathrm{b}, *}$, Zhansi Jiang ${ }^{\mathrm{a}}$ and Xuefeng Chen ${ }^{\mathrm{b}}$ \\ ${ }^{a}$ School of Mechantronic Engineering, Guilin University of Electronic Technology, Guilin, 541004, P.R. China \\ ${ }^{\mathrm{b}}$ State Key Laboratory for Manufacturing Systems Engineering, Xi'an Jiaotong University, Xi'an, 710049, P.R. \\ China
}

Received 6 June 2009

Accepted 8 October 2009

\begin{abstract}
A class of wavelet-based Rayleigh-Euler rotating beam element using B-spline wavelets on the interval (BSWI) is developed to analyze rotor-bearing system. The effects of translational and rotary inertia, torsion moment, axial displacement, cross-coupled stiffness and damping coefficients of bearings, hysteric and viscous internal damping, gyroscopic moments and bending deformation of the system are included in the computational model. In order to get a generalized formulation of waveletbased element, each boundary node is collocated six degrees of freedom (DOFs): three translations and three rotations; whereas, each inner node has only three translations. Typical numerical examples are presented to show the accuracy and efficiency of the presented method.
\end{abstract}

Keywords: Finite element method, wavelet-based element, shafts, internal damping, dynamic analysis

\section{Introduction}

A recent trend in modern rotating machinery design has been the evolution of higher rotating speeds and lower weight of rotating components, such as shaft, turbine blades, aircraft propeller blades and drill bits, etc. This trend has been accompanied by a more accurate numerical analysis method to predict dynamic behaviors of rotating structures. Recently, there were a number of studies relating to this field in the past decades as indicated in the book by Ehrich [1] and in the survey paper by Meng [2] and Huang et al. [3]. Numerous researchers focused their interest on the field of analyzing the dynamic characteristics of rotating structures, which are concerned with determining critical speeds, whirl speeds (natural frequencies), instability thresholds and unbalance response.

High precision numerical approximations have been developed to analyze the dynamic behavior of rotating structures by using finite element method. A rotating shaft element using Timoshenko beam theory was proposed by Nelson [4] and Greenhill et al. [5]. The computational model for rotor-bearing systems to compute natural whirl speeds and instability thresholds were investigated by Zorzi and Nelson [6], Ku [7], Kalita and Kakoty [8]. Those works showed that the use of finite elements for the modeling of rotor-bearing systems makes it possible to formulate increasingly complicated problems and to yield highly accurate and successful results. However, the above mentioned literatures employed the conventional finite element method. In order to gain accurate results, numerous elements are needed. In order to promote the analysis precision and efficiency, Hashemi et al. developed a new dynamic finite element (DFE) formulation for the vibration analysis of spinning beams [9]. Wang et al. proposed a novel beam finite element having two nodes and 16 degrees of freedom to analyze free vibration characters of

\footnotetext{
*Corresponding author. E-mail: wxw8627@163.com.
} 
stepped shafts [10]. Banerjee and Su developed a dynamic stiffness matrix for free vibration analysis of spinning beams $[11,12]$.

Unlike conventional finite element method, the desirable advantages of wavelet-based elements are various basis functions for structural analysis [13,14]. By means of "two-scale relations" of wavelets, the scale adopted can be changed freely according to requirements to improve analysis accuracy. Xiang et al. constructed some classes of 1D and 2D BSWI elements for structural analysis with high performance [15-18]. In addition, Han et al. constructed some spline wavelet elements for analyzing structural mechanics problems under the theory frame of spline elements [19]. Xiang et al. also presented the BSWI elements to detect cracks in beam and shaft with high precisions [20,21]. However, the wavelet-based rotating beam element that considers internal damping was not constructed in the published literatures.

Although the effects of various factors on the dynamic characteristics of rotor-bearing systems have been investigated via the improved finite element model, a common feature of the published works is that those finite elements belong to conventional finite element method. The object of the present article is to develop a class of high performance of BSWI Rayleigh-Euler beam elements to analyze spinning structures in engineering, especially complex rotor-bearing systems. In the present finite element model, each element has a certain nodes according to the level of wavelet basis. Each boundary node has six degrees of freedom (DOFs): three translations and three rotations, whereas each inner node has only three translations.

The outline of this paper is as follows. In Sections 2, a class of novel BSWI rotating Rayleigh-Euler shaft elements is constructed. The elements include the effects of translational and rotary inertia and the gyroscopic moments, the combined effects of bending deformations and the internal viscous and hysteretic damping, cross-coupled stiffness and damping coefficients of bearings, In Section 3, some numerical studies are made by compared with the other previously published works to investigate the present wavelet-based shaft element.

\section{BSWI beam element formulation}

\subsection{The construction of BSWI rotating Rayleigh-Euler beam elements}

Classical approaches to wavelet construction deal with multi-resolution analysis (MRA) on the whole real space $\mathbf{R}$ and the corresponding wavelets are often defined on the whole square integrable real space $L\left(\mathbf{R}^{2}\right)$. Sometimes numerical instability phenomenon will be occurred when this kind of wavelets is applied to numerical simulation of partial differential equations (PDEs) [22]. To overcome this limitation, Chui and Quak constructed BSWI functions, and presented a decomposition and reconstruction algorithm [23]. The scaling functions $\phi_{m, k}^{j}(\xi)$ for order $m$ at the scale $j$ are simply denoted as BSWI $m_{j}$ scaling functions $\Phi_{m j}$, i.e.

$$
\Phi_{m j}=\left\{\phi_{m,-m+1}^{j}(\xi) \phi_{m,-m+2}^{j}(\xi) \ldots \phi_{m, 2^{j}-1}^{j}(\xi)\right\} .
$$

The explicit expression of each term $\phi_{m, k}^{j}(\xi)$ for order $m$ at the scale $j$ is shown in [17].

The slender shaft is modeled by a Rayleigh-Euler beam considering the effects of the cross-section inertia, torsion moment and axial displacement, the element potential energy $U^{e}$ can be written as

$$
U^{e}=\frac{1}{2} \int_{0}^{l_{e}} E I_{z}\left(\frac{\mathrm{d}^{2} w}{\mathrm{~d} x^{2}}\right)^{2} \mathrm{~d} x+\frac{1}{2} \int_{0}^{l_{e}} E I_{y}\left(\frac{\mathrm{d}^{2} v}{\mathrm{~d} x^{2}}\right)^{2} \mathrm{~d} x+\frac{1}{2} \int_{0}^{l_{e}} G J_{x}\left(\frac{\mathrm{d} \theta_{x}}{\mathrm{~d} x}\right)^{2} \mathrm{~d} x+\frac{1}{2} \int_{0}^{l_{e}} E A\left(\frac{d u}{\mathrm{~d} x}\right)^{2} \mathrm{~d} x,(2)
$$

where $E$ is the Young's modulus, $I_{z}$ and $I_{y}$ are the moment of inertia, $J_{x}$ is the polar moment of inertia. $w(x, t)$ and $v(x, t)$ are the transverse displacement, $u(x, t)$ is the axial displacement, $\theta_{x}(x, t)$ is the rotation of torsion, $l_{e}$ is the element length, $A$ is the cross-section area, $G$ is the shear modulus.

The element kinetic energy $T^{e}$ of the Rayleigh-Euler beam allowing for the rotatory inertia effect, including the translational and rotational forms, is given by

$$
\begin{aligned}
T^{e}= & \frac{1}{2} \int_{0}^{l_{e}} \rho A\left[\left(\frac{\partial w}{\partial t}\right)^{2}+\left(\frac{\partial v}{\partial t}\right)^{2}\right] \mathrm{d} x+\frac{1}{2} \int_{0}^{l_{e}} \rho I_{Z}\left(\frac{\partial \theta_{Z}}{\partial t}\right)^{2} \mathrm{~d} x+\frac{1}{2} \int_{0}^{l_{e}} \rho I_{y}\left(\frac{\partial \theta_{y}}{\partial t}\right)^{2} \mathrm{~d} x \\
& +\frac{\Omega J_{x} \rho}{2} \int_{0}^{l_{e}}\left[\theta_{z}\left(\frac{\partial \theta_{y}}{\partial t}\right)^{2}-\theta_{y}\left(\frac{\partial \theta_{z}}{\partial t}\right)^{2}\right] \mathrm{d} x+\frac{1}{2} \int_{0}^{l_{e}} \rho I_{x}\left(\frac{\partial \theta_{x}}{\partial t}\right)^{2} \mathrm{~d} x+\frac{1}{2} \int_{0}^{l_{e}} \rho A\left(\frac{\partial u}{\partial t}\right)^{2} \mathrm{~d} x
\end{aligned}
$$




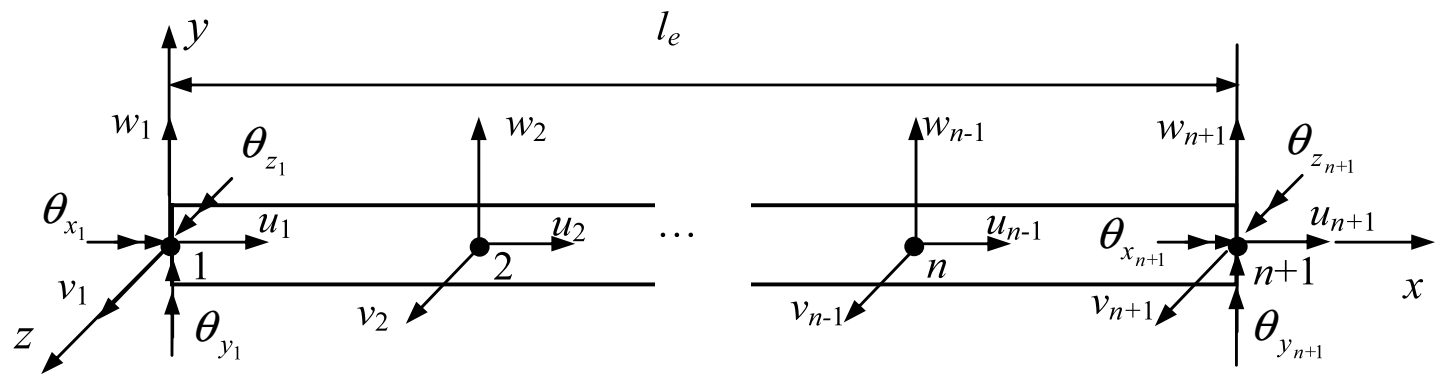

Fig. 1. The layout of element nodes and the corresponding DOFs for wavelet-based element.

where $\rho$ is the density, $\Omega$ is the rotational speed $(\mathrm{rad} / \mathrm{s}), \theta_{z}(x, t)$ and $\theta_{y}(x, t)$ are the rotation of the beam section due to bending and can be given by

$$
\left\{\begin{array}{l}
\theta_{z}=\frac{\mathrm{d} w}{\mathrm{~d} x}=\frac{1}{l_{e}} \frac{\mathrm{d} w}{\mathrm{~d} \xi} \\
\theta_{y}=\frac{\mathrm{d} v}{\mathrm{~d} x}=\frac{1}{l_{e}} \frac{\mathrm{d} v}{\mathrm{~d} \xi}
\end{array} .\right.
$$

In order to satisfy the displacement and slope compatibility among neighboring elements, the element boundary nodes should include the transverse displacements and slopes [16]. In the present study, the transverse displacements are interpolated by BSWI $4_{j}$ scaling functions $\Phi_{4 j}$, the axial displacement is interpolated by BSWI $2_{j}$ scaling functions $\Phi_{2 j}$, and the rotation of torsion is interpolated by conventional linear interpolation. Therefore, element degrees of freedom (DOFs) on each boundary node in physical space include three displacements and three slopes, i.e. $u_{i}, v_{i}, w_{i}, \theta_{x_{i}}, \theta_{y_{i}}, \theta_{z_{i}}(i=1, n+1)$. While on each inner node, it only have three displacements, i.e. $u_{i}, v_{i}, w_{i}(i=$ $2,3, \cdots, n$ ). In this study, the beam element is divided for solving domain $\Omega_{e}$ into $n=2^{j}$ ( $j$ is the scale of BSWI) segments, the node number is $n+1$, the element degrees of freedom (DOFs) $=3 \times 2^{j}+9$. The layout of element nodes is shown in Fig. 1. The element is abbreviated to BSWI $m_{j}$ Rayleigh-Euler rotating beam element.

The element physical DOFs can be represented by

$$
\delta^{e}=\left\{u_{1} v_{1} w_{1} \theta_{x_{1}} \theta_{y_{1}} \theta_{z_{1}} u_{2} v_{2} w_{2} \cdots u_{n} v_{n} w_{n} u_{n+1} v_{n+1} w_{n+1} \theta_{x_{n+1}} \theta_{y_{n+1}} \theta_{z_{n+1}}\right\}^{T},
$$

where $\theta_{z_{1}}=\frac{1}{l_{e}} \frac{\mathrm{d} w_{1}}{\mathrm{~d} \xi}, \theta_{y_{1}}=\frac{1}{l_{e}} \frac{\mathrm{d} v_{1}}{\mathrm{~d} \xi}, \theta_{z_{n+1}}=\frac{1}{l_{e}} \frac{\mathrm{d} w_{n+1}}{\mathrm{~d} \xi}$ and $\theta_{y_{n+1}}=\frac{1}{l_{e}} \frac{\mathrm{d} v_{n+1}}{\mathrm{~d} \xi}$ denote rotation on each element endpoint.

The unknown field functions $v(\xi, t)$ and $w(\xi, t)$ are interpolated by $\Phi_{4 j}$ as

$$
\left\{\begin{array}{l}
w(\xi, t)=\Phi_{4 j} \mathbf{T}_{b}^{e} \mathbf{w}^{e} \\
v(\xi, t)=\Phi_{4 j} \mathbf{T}_{b}^{e} \mathbf{v}^{e}
\end{array}\right.
$$

where transformation matrix $\mathbf{T}_{b}^{e}$ is given by [16], as

$$
\mathbf{T}_{b}^{e}=\left(\left[\Phi_{4 j}^{T}\left(\xi_{1}\right) \frac{1}{l_{e}} \frac{d \Phi_{4 j}^{T}\left(\xi_{1}\right)}{d \xi} \Phi_{4 j}^{T}\left(\xi_{2}\right) \ldots \Phi_{4 j}^{T}\left(\xi_{n}\right) \Phi_{4 j}^{T}\left(\xi_{n+1}\right) \frac{1}{l_{e}} \frac{d \Phi_{4 j}^{T}\left(\xi_{n+1}\right)}{d \xi}\right]^{T}\right)^{-1}
$$

and physical DOFs vector $\mathbf{w}^{e}$ and $\mathbf{v}^{e}$ are given by

$$
\left\{\begin{array}{l}
\mathbf{w}^{e}=\left\{w_{1} \theta_{z_{1}} w_{2} w_{3} \cdots w_{n} w_{n+1} \theta_{z_{n+1}}\right\}^{T} \\
\mathbf{v}^{e}=\left\{v_{1} \theta_{y_{1}} v_{2} v_{3} \cdots v_{n} v_{n+1} \theta_{y_{n+1}}\right\}^{T}
\end{array} .\right.
$$

The unknown field function $u(\xi, t)$ is interpolated by $\Phi_{2 j}$ as

$$
u(\xi, t)=\Phi_{2 j} \mathbf{T}_{a}^{e} \mathbf{u}^{e},
$$

where transformation matrix $\mathbf{T}_{a}^{e}$ is given by [16], as 


$$
\mathbf{T}_{a}^{e}=\left(\left[\Phi_{2 j}^{T}\left(\xi_{1}\right) \Phi_{2 j}^{T}\left(\xi_{2}\right) \ldots \Phi_{2 j}^{T}\left(\xi_{n}\right) \Phi_{2 j}^{T}\left(\xi_{n+1}\right)\right]^{T}\right)^{-1},
$$

and physical DOFs vector $\mathbf{u}^{e}$ is given by

$$
\mathbf{u}^{e}=\left\{u_{1} u_{2} \cdots u_{n} u_{n+1}\right\}^{T} .
$$

The unknown field function $\theta_{x}(x, t)$ is interpolated by conventional linear bases as

$$
\theta_{x}(x, t)=\mathbf{N} \theta_{x}^{e},
$$

where

$$
\mathbf{N}=\left\{N_{1} N_{2}\right\},
$$

where

$$
\left\{\begin{array}{l}
N_{1}=1-\xi \\
N_{2}=\xi
\end{array}\right.
$$

and physical DOFs vector $\theta_{x}^{e}$ is given by

$$
\theta_{x}^{e}=\left\{\theta_{x_{1}} \theta_{x_{n+1}}\right\}^{T} .
$$

Substitution Eqs (6), (9) and (11) into Eqs (2) and (3), respectively, we obtain

$$
\begin{aligned}
U^{e}= & \frac{1}{2}\left(\mathbf{w}^{e}\right)^{T} \mathbf{K}_{b y}^{e}\left(\mathbf{w}^{e}\right)+\frac{1}{2}\left(\mathbf{v}^{e}\right)^{T} \mathbf{K}_{b z}^{e}\left(\mathbf{v}^{e}\right)+\frac{1}{2}\left(\theta_{x}^{e}\right)^{T} \mathbf{K}_{t x}^{e}\left(\theta_{x}^{e}\right)+\frac{1}{2}\left(\mathbf{u}^{e}\right)^{T} \mathbf{K}_{a x}^{e}\left(\mathbf{u}^{e}\right), \\
T^{e}= & \frac{1}{2}\left(\frac{\partial \mathbf{w}^{e}}{\partial t}\right)^{T} \mathbf{M}_{b y}^{e}\left(\frac{\partial \mathbf{w}^{e}}{\partial t}\right)+\frac{1}{2}\left(\frac{\partial \mathbf{v}^{e}}{\partial t}\right)^{T} \mathbf{M}_{b z}^{e}\left(\frac{\partial \mathbf{v}^{e}}{\partial t}\right)+\frac{1}{2}\left(\frac{\partial \mathbf{w}^{e}}{\partial t}\right)^{T} \mathbf{M}_{r y}^{e}\left(\frac{\partial \mathbf{w}^{e}}{\partial t}\right) \\
& +\frac{1}{2}\left(\frac{\partial \mathbf{v}^{e}}{\partial t}\right)^{T} \mathbf{M}_{r z}^{e}\left(\frac{\partial \mathbf{v}^{e}}{\partial t}\right)+\frac{1}{2}\left(\frac{\partial \mathbf{w}^{e}}{\partial t}\right)^{T} \mathbf{G}^{e}\left(\mathbf{v}^{e}\right)-\frac{1}{2}\left(\frac{\partial \mathbf{v}^{e}}{\partial t}\right)^{T} \mathbf{G}^{e}\left(\mathbf{w}^{e}\right), \\
& +\frac{1}{2}\left(\frac{\partial \theta_{x}^{e}}{\partial t}\right)^{T} \mathbf{M}_{t x}^{e}\left(\frac{\partial \theta_{x}^{e}}{\partial t}\right)+\frac{1}{2}\left(\frac{\partial \mathbf{u}^{e}}{\partial t}\right)^{T} \mathbf{M}_{a x}^{e}\left(\frac{\partial \mathbf{u}^{e}}{\partial t}\right)
\end{aligned}
$$

where the element bending stiffness matrices $\mathbf{K}_{b y}^{e}$ and $\mathbf{K}_{b z}^{e}$, torsion stiffness matrix $\mathbf{K}_{t x}^{e}$ and axial stiffness matrix $\mathbf{K}_{a x}^{e}$ are

$$
\begin{aligned}
& \mathbf{K}_{b y}{ }^{e}=\frac{E I_{z}}{l_{e}{ }^{3}}\left(\mathbf{T}_{b}{ }^{e}\right)^{T} \Gamma^{2,2} \mathbf{T}_{b}{ }^{e}, \\
& \mathbf{K}_{b z}^{e}=\frac{E I_{y}}{l_{e}^{3}}\left(\mathbf{T}_{b}^{e}\right)^{T} \Gamma^{2,2} \mathbf{T}_{b}^{e}, \\
& \mathbf{K}_{t x}^{e}=\frac{G J_{x}}{l_{e}} \mathbf{A}^{1,1}, \\
& \mathbf{K}_{a x}^{e}=\frac{E A}{l_{e}}\left(\mathbf{T}_{a}^{e}\right)^{T} \Gamma_{1}^{1,1} \mathbf{T}_{a}^{e},
\end{aligned}
$$

the element translational mass matrices $\mathbf{M}_{b y}^{e}, \mathbf{M}_{b z}^{e}$ and $\mathbf{M}_{a x}^{e}$ are

$$
\begin{aligned}
& \mathbf{M}_{b y}^{e}=\mathbf{M}_{b z}^{e}=\rho A l_{e}\left(\mathbf{T}_{b}^{e}\right)^{T} \Gamma^{0,0} \mathbf{T}_{b}^{e}, \\
& \mathbf{M}_{a x}^{e}=\rho A l_{e}\left(\mathbf{T}_{a}^{e}\right)^{T} \Gamma_{1}^{0,0} \mathbf{T}_{a}^{e},
\end{aligned}
$$

the element rotatory inertia mass matrices $\mathbf{M}_{r y}^{e}$ and $\mathbf{M}_{r z}^{e}$ are

$$
\mathbf{M}_{r y}^{e}=\frac{\rho I_{z}}{l_{e}}\left(\mathbf{T}_{b}^{e}\right)^{T} \Gamma^{1,1} \mathbf{T}_{b}^{e},
$$




$$
\mathbf{M}_{r z}^{e}=\frac{\rho I_{y}}{l_{e}}\left(\mathbf{T}_{b}^{e}\right)^{T} \Gamma^{1,1} \mathbf{T}_{b}^{e}
$$

the element torsion mass matrix $\mathbf{M}_{t x}^{e}$ is

$$
\mathbf{M}_{t x}^{e}=\rho J_{x} l_{e} \mathbf{A}^{0,0}
$$

and the element gyroscopic matrix $\mathbf{G}^{e}$ is

$$
\mathbf{G}^{e}=\frac{\Omega J_{x} \rho}{l_{e}}\left(\mathbf{T}_{b}^{e}\right)^{T} \Gamma^{1,1} \mathbf{T}_{b}^{e}
$$

where

$$
\begin{aligned}
\Gamma^{2,2} & =\int_{0}^{1} \frac{\mathrm{d}^{2} \Phi_{4 j}^{T}}{\mathrm{~d} \xi^{2}} \frac{\mathrm{d}^{2} \Phi_{4 j}}{\mathrm{~d} \xi^{2}} \mathrm{~d} \xi \\
\Gamma^{1,1} & =\int_{0}^{1} \frac{\mathrm{d} \Phi_{4 j}^{T}}{\mathrm{~d} \xi} \frac{\mathrm{d} \Phi_{4 j}}{\mathrm{~d} \xi} \mathrm{d} \xi \\
\Gamma^{0,0} & =\int_{0}^{1} \Phi_{4 j}^{T} \Phi_{4 j} \mathrm{~d} \xi \\
\Gamma_{1}^{1,1} & =\int_{0}^{1} \frac{\mathrm{d} \Phi_{2 j}^{T}}{\mathrm{~d} \xi} \frac{\mathrm{d} \Phi_{2 j}}{\mathrm{~d} \xi} \mathrm{d} \xi \\
\Gamma_{1}^{0,0} & =\int_{0}^{1} \Phi_{2 j}^{T} \Phi_{2 j} \mathrm{~d} \xi \\
\boldsymbol{A}^{1,1} & =\int_{0}^{1} \frac{\mathrm{d} \mathbf{N}^{T}}{\mathrm{~d} \xi} \frac{\mathrm{d} \boldsymbol{N}}{\mathrm{d} \xi} \mathrm{d} \xi \\
\boldsymbol{A}^{0,0} & =\int_{0}^{1} \boldsymbol{N}^{T} \boldsymbol{N} \mathrm{d} \xi .
\end{aligned}
$$

Applying Hamilton's principle, the element free vibration equation can be obtained as

$$
\begin{aligned}
& {\left[\begin{array}{ccccc}
\mathbf{M}_{b y}^{e}+\mathbf{M}_{r y}^{e} & 0 & 0 & 0 \\
0 & \mathbf{M}_{b z}^{e}+\mathbf{M}_{r z}^{e} & 0 & 0 \\
0 & 0 & \mathbf{M}_{t x}^{e} & 0 \\
0 & & 0 & 0 & \mathbf{M}_{a x}^{e}
\end{array}\right]\left[\begin{array}{c}
\frac{\partial^{2} \mathbf{w}^{e}}{\partial t^{2}} \\
\frac{\partial^{2} \mathbf{v}^{e}}{\partial t^{2}} \\
\frac{\partial^{2} \mathbf{u}^{e}}{\partial t^{2}} \\
\frac{\partial^{2} \theta_{x}^{e}}{\partial t^{2}}
\end{array}\right]+\left[\begin{array}{cccc}
0 & \boldsymbol{G}^{e} & 0 & 0 \\
-\boldsymbol{G}^{e} & 0 & 0 & 0 \\
0 & 0 & 0 & 0 \\
0 & 0 & 0 & 0
\end{array}\right]\left[\begin{array}{c}
\frac{\partial \mathbf{w}^{e}}{\partial t} \\
\frac{\partial \mathbf{v}^{e}}{\partial t} \\
\frac{\partial \mathbf{u}^{e}}{\partial t} \\
\frac{\partial \theta_{x}^{e}}{\partial t}
\end{array}\right] .} \\
& +\left[\begin{array}{cccc}
\mathbf{K}_{b y}^{e} & 0 & 0 & 0 \\
0 & \mathbf{K}_{b z}^{e} & 0 & 0 \\
0 & 0 & \mathbf{K}_{a x}^{e} & 0 \\
0 & 0 & 0 & \mathbf{K}_{t x}^{e}
\end{array}\right]\left[\begin{array}{c}
\mathbf{w}^{e} \\
\mathbf{v}^{e} \\
\mathbf{u}^{e} \\
\theta_{x}^{e}
\end{array}\right]=0
\end{aligned}
$$

Rewrite Eq. (29) according to the layout of element physical DOF as mentioned on Eq. (5), we have

$$
\overline{\mathbf{M}}^{e} \frac{\partial^{2} \delta^{e}}{\partial t^{2}}+\overline{\mathbf{G}}^{e} \frac{\partial \delta^{e}}{\partial t}+\overline{\mathbf{K}}^{e} \delta^{e}=0
$$

where $\overline{\mathbf{M}}^{e}, \overline{\mathbf{G}}^{e}$ and $\overline{\mathbf{K}}^{e}$ are the element mass matrix, element gyroscopic matrix and element stiffness matrix that rearrange from Eq. (29) according to Eq. (5) by elementary transformation. 


\subsection{Incorporation of internal damping}

As all real materials have the capability of dissipating mechanical energy, it is important that, for high-speed operation, the internal damping is necessary to be considered in the finite element model of rotor-bearing systems. Zorzi and Nelson [6] considered the combined effects of both viscous and hysteretic internal damping in their finite element formulation of the rotor-bearing system. Ku extended the internal damping model to whirl speed and stability of Rayleigh-Timoshenko shafts [7]. Greenhill et al. also employed the internal damping model to construct conical beam element for rotor dynamic analysis [24]. Using $\eta_{V}$ and $\eta_{H}$ to denote the viscous damping coefficient and the hysteretic loss factor of the shaft material, according to the former studies, vibration equation can be expressed as

$$
\overline{\mathbf{M}}^{e} \frac{\partial^{2} \delta^{e}}{\partial t^{2}}+\left(\eta_{V} \overline{\mathbf{K}}_{c 1}^{e}+\overline{\mathbf{G}}^{e}\right) \frac{\partial \delta^{e}}{\partial t}+\left(\eta_{a} \overline{\mathbf{K}}^{e}+\eta_{b} \overline{\mathbf{K}}_{c 2}^{e}\right) \delta^{e}=\overline{\mathbf{F}}^{e}
$$

where

$$
\left\{\begin{array}{l}
\eta_{a}=\frac{1+\eta_{H}}{\sqrt{1+\eta_{H}^{2}}} \\
\eta_{b}=\frac{\eta_{H}}{\sqrt{1+\eta_{H}^{2}}}+\Omega \eta_{V}
\end{array},\right.
$$

and matrices $\mathbf{K}_{c 1}^{e}$ and $\mathbf{K}_{c 2}^{e}$ are

$$
\left\{\begin{array}{l}
\mathbf{K}_{c 1}^{e}=\overline{\mathbf{R}}_{1} \overline{\mathbf{K}}^{e} \\
\mathbf{K}_{c 2}^{e}=\overline{\mathbf{R}}_{2} \overline{\mathbf{K}}^{e},
\end{array}\right.
$$

where the transformation matrix $\overline{\mathbf{R}}_{1}$ is defined by

$$
\overline{\mathbf{R}}_{1}=\left[\begin{array}{llllll}
\mathbf{R}_{1}^{1} & & & & & \\
& \mathbf{R}_{1} & & & & \\
& & \mathbf{R}_{1} & & & \\
& & & \ddots & & \\
& & & & \mathbf{R}_{1} & \\
& & & & & \mathbf{R}_{1}^{1}
\end{array}\right]_{\left(3 \times 2^{j}+9\right) \times\left(3 \times 2^{j}+9\right)},
$$

where

$$
\mathbf{R}_{1}^{1}=\left[\begin{array}{cccccc}
0 & 0 & 0 & 0 & 0 & 0 \\
0 & 1 & 0 & 0 & 0 & 0 \\
0 & 0 & -1 & 0 & 0 & 0 \\
0 & 0 & 0 & 0 & 0 & 0 \\
0 & 0 & 0 & 0 & 1 & 0 \\
0 & 0 & 0 & 0 & 0 & -1
\end{array}\right] \text { and } \mathbf{R}_{1}=\left[\begin{array}{ccc}
0 & 0 & 0 \\
0 & 1 & 0 \\
0 & 0 & -1
\end{array}\right]
$$

the transformation matrix $\overline{\mathbf{R}}_{2}$ is defined by

$$
\overline{\mathbf{R}}_{2}=\left[\begin{array}{cccccc}
\mathbf{R}_{2}^{1} & 0 & 0 & 0 & 0 & 0 \\
0 & \mathbf{R}_{2} & 0 & 0 & 0 & 0 \\
0 & 0 & \mathbf{R}_{2} & 0 & 0 & 0 \\
0 & 0 & 0 & \ddots & 0 & 0 \\
0 & 0 & 0 & 0 & \mathbf{R}_{2} & 0 \\
0 & 0 & 0 & 0 & 0 & \mathbf{R}_{2}^{1}
\end{array}\right]_{\left(3 \times 2^{j}+9\right) \times\left(3 \times 2^{j}+9\right)}
$$

where 


$$
\mathbf{R}_{2}^{1}=\left[\begin{array}{cccccc}
0 & 0 & 0 & 0 & 0 & 0 \\
0 & 0 & 1 & 0 & 0 & 0 \\
0 & -1 & 0 & 0 & 0 & 0 \\
0 & 0 & 0 & 0 & 0 & 0 \\
0 & 0 & 0 & 0 & 0 & 1 \\
0 & 0 & 0 & 0 & -1 & 0
\end{array}\right] \text { and } \mathbf{R}_{2}=\left[\begin{array}{ccc}
0 & 0 & 0 \\
0 & 0 & 1 \\
0 & -1 & 0
\end{array}\right]
$$

and $\overline{\mathbf{F}}^{e}$ is the vector of exciting forces.

\subsection{Bearings}

The classic linearized model with eight spring and damping coefficients is employed for the modeling of bearing in the present work. The forces at each bearing are assumed to obey the governing equations of the following form

$$
\overline{\mathbf{C}}^{b} \frac{\partial \boldsymbol{\delta}_{b}^{e}}{\partial t}+\overline{\mathbf{K}}^{b} \boldsymbol{\delta}_{b}^{e}=\overline{\mathbf{F}}^{b}
$$

where $\overline{\mathbf{F}}^{b}$ is the vector of the bearing forces, $\delta_{b}^{e}$ is the vector of bearing DOFs, the bearing damping matrix $\overline{\mathbf{C}}^{b}$ and stiffness matrix $\overline{\mathbf{K}}^{b}$ are

$$
\left\{\begin{array}{l}
\overline{\mathbf{C}}^{b}=\left[\begin{array}{ll}
C_{w w} & C_{w v} \\
C_{v w} & C_{v v}
\end{array}\right] \\
\overline{\mathbf{K}}^{b}=\left[\begin{array}{ll}
K_{w w} & K_{w v} \\
K_{v w} & C_{v v}
\end{array}\right],
\end{array}\right.
$$

where $C_{i j}$ and $K_{i j}$ are the bearing damping and stiffness coefficients.

\subsection{Discs}

The discrete disc, which is thin and symmetric about the axis of rotation, has the following form of governing equations

$$
\overline{\mathbf{M}}^{d} \frac{\partial^{2} \delta_{d}^{e}}{\partial t^{2}}+\overline{\mathbf{G}}^{d} \frac{\partial \delta_{d}^{e}}{\partial t}=\overline{\mathbf{F}}^{d}
$$

where $\overline{\mathbf{F}}^{d}$ is the $6 \times 1$ vector of the exciting forces, $\delta_{d}^{e}$ is the $6 \times 1$ vector of rigid disc DOFs, the disc gyroscopic matrix $\overline{\mathbf{G}}^{d}$ and mass matrix $\overline{\mathbf{M}}^{d}$ are defined by

$$
\begin{aligned}
\overline{\mathbf{G}}^{d}= & {\left[\begin{array}{cccccc}
0 & 0 & 0 & 0 & 0 & 0 \\
0 & 0 & 0 & 0 & 0 & 0 \\
0 & 0 & 0 & 0 & 0 & -J_{x}^{d} \\
0 & 0 & 0 & 0 & 0 & 0 \\
0 & 0 & 0 & 0 & 0 & 0 \\
0 & 0 & J_{x}^{d} & 0 & 0 & 0
\end{array}\right], } \\
\overline{\mathbf{M}}^{d}= & {\left[\begin{array}{cccccc}
0 & 0 & 0 & 0 & 0 & 0 \\
0 & J_{d} & 0 & 0 & 0 & 0 \\
0 & 0 & J_{x}^{d} & 0 & 0 & 0 \\
0 & 0 & 0 & 0 & 0 & 0 \\
0 & 0 & 0 & 0 & J_{d} & 0 \\
0 & 0 & 0 & 0 & 0 & J_{x}^{d}
\end{array}\right] }
\end{aligned}
$$

where the rigid disc diametrical and polar mass moments of inertia are

$$
\left\{\begin{array}{l}
J_{d}=\frac{m_{d}}{4}\left(r_{d}^{2}+\frac{h_{d}^{2}}{3}\right) \\
J_{x}^{d}=\frac{m_{d} r_{d}^{2}}{2}
\end{array},\right.
$$

where $h_{d}$ is the disc thickness, $r_{d}$ is the disc radius and $m_{d}$ is the disc mass. 


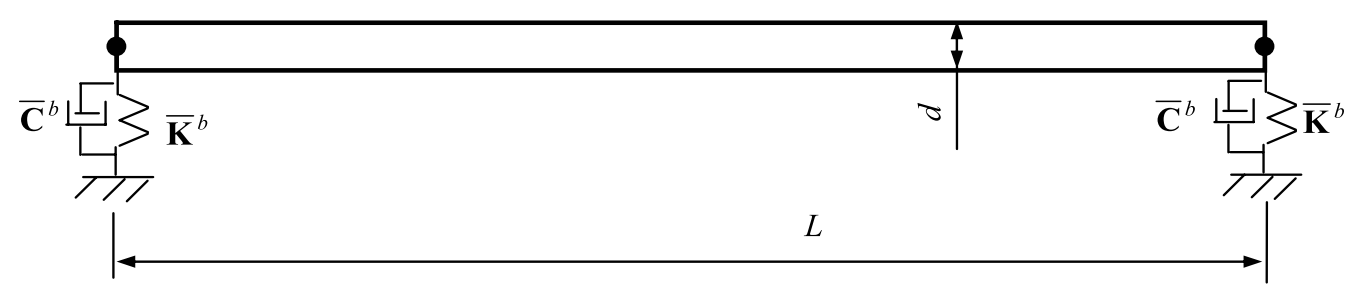

Fig. 2. Rotor-bearing systems.

\subsection{System equation of motion}

The equations of motion of the complete system can be obtained by assembling element vibration equation, that is

$$
\overline{\mathbf{M}} \frac{\partial^{2} \boldsymbol{\delta}}{\partial t^{2}}+\overline{\mathbf{G}} \frac{\partial \boldsymbol{\delta}}{\partial t}+\overline{\mathbf{K}} \delta=\mathbf{F}
$$

where $\overline{\mathbf{M}}, \overline{\mathbf{G}}, \overline{\mathbf{K}}$ and $\mathbf{F}$ are system mass matrix, gyroscopic and damping matrix, stiffness matrix and exciting force, respectively.

For the analysis of natural whirl speeds and instability thresholds of the rotor-bearing system, the force term can be omitted. The right hand of system equations of the motion then is set to zeros.

Neglecting the exciting force, Eq. (44) is written in the first order state vector form as

$$
\boldsymbol{E} \frac{\partial \boldsymbol{q}}{\partial t}+\boldsymbol{F} q=0
$$

where

$$
\begin{aligned}
& \boldsymbol{q}=\left[\begin{array}{c}
\frac{\partial \boldsymbol{\delta}}{\partial t} \\
\boldsymbol{\delta}
\end{array}\right], \\
& \mathbf{E}=\left[\begin{array}{cc}
0 & -\overline{\mathbf{M}} \\
\overline{\mathbf{M}} & \overline{\mathbf{G}}
\end{array}\right], \\
& \mathbf{F}=\left[\begin{array}{cc}
\overline{\mathbf{M}} & 0 \\
0 & \overline{\mathbf{K}}
\end{array}\right] .
\end{aligned}
$$

The associated eigenvalue problem for Eq. (45) is sought from an assumed solution form as

$$
\boldsymbol{q}=\boldsymbol{q}_{0} e^{\lambda t}
$$

Substituting Eq. (49) into Eq. (46), the global free vibration frequency equations are given by

$$
|\mathbf{E} \lambda+\mathbf{F}|=0
$$

where $\lambda=\sigma+i \cdot \omega=\sigma+i \cdot 2 \pi f$ is the complex eigenvalue. $\omega(\mathrm{rad} / \mathrm{s})$ is the natural whirl speed, $f(\mathrm{~Hz})$ is the modal frequency of structural dynamic systems. $\sigma$ represents the instability threshold when $\sigma>0$. The parameter of logarithmic decrement $\delta$ is defined as

$$
\delta=-\frac{2 \pi \sigma}{\omega}
$$

where $\delta$ represents the instability threshold when $\delta<0$. 
Table 1

Whirl speeds in $\mathrm{rad} / \mathrm{s}$ of a uniform shaft with isotropic undamped flexible bearings at a spin speed of $4000 \mathrm{rpm}$

\begin{tabular}{|c|c|c|c|c|c|c|c|c|}
\hline \multirow[b]{2}{*}{ Mode } & \multicolumn{4}{|c|}{$\eta_{H}=0.0002$} & \multicolumn{4}{|c|}{$\eta_{V}=0.0002 \mathrm{~s}$} \\
\hline & Present & Ref. [7] & Ref. [8] & Ref. [25] & Present & Ref. [7] & Ref. [8] & Ref. [25] \\
\hline $1 \mathrm{~F}$ & 521.01 & 519.78 & 520.0 & 520.10 & 522.02 & 521.48 & 520.0 & 521.79 \\
\hline $1 \mathrm{~B}$ & 520.46 & 519.23 & 519.4 & 519.54 & 521.67 & 519.75 & 519.4 & 520.06 \\
\hline $2 \mathrm{~F}$ & 1096.52 & 1094.40 & 1094.8 & 1095.28 & 1097.55 & 1095.13 & 1094.8 & 1096.01 \\
\hline $2 \mathrm{~B}$ & 1092.99 & 1090.90 & 1091.6 & 1091.77 & 1096.26 & 1094.52 & 1091.6 & 1095.34 \\
\hline $3 F$ & 2251.47 & 2238.53 & 2241.9 & 2244.72 & 2222.56 & 2216.81 & 2241.8 & 2222.78 \\
\hline $3 B$ & 2236.25 & 2223.80 & 2228.0 & 2229.82 & 2219.83 & 2201.25 & 2227.9 & 2206.94 \\
\hline $4 \mathrm{~F}$ & 5077.05 & 4968.16 & 4987.7 & 5020.12 & 4463.13 & 4413.32 & 4987.2 & 4447.40 \\
\hline 4B & 5041.94 & 4935.91 & 4954.1 & 4986.74 & 4459.85 & 4378.95 & 4933.6 & 4411.81 \\
\hline
\end{tabular}

Table 2

Logarithmic decrement $\delta$ of a uniform shaft with isotropic undamped bearings at a spin speed of $4000 \mathrm{rpm}$ (suppose $\left.\eta_{H}=0.0002\right)$

\begin{tabular}{crrrr}
\hline Mode & \multicolumn{1}{c}{ Present } & \multicolumn{1}{c}{ Ref. [6] } & \multicolumn{1}{c}{ Ref. [7] } & \multicolumn{1}{c}{ Ref. [25] } \\
\hline 1F & $-2.48 \times 10^{-4}$ & $-2.48 \times 10^{-4}$ & $-2.85 \times 10^{-4}$ & $-2.49 \times 10^{-4}$ \\
1B & $2.50 \times 10^{-4}$ & $2.50 \times 10^{-4}$ & $2.87 \times 10^{-4}$ & $2.51 \times 10^{-4}$ \\
2F & $-4.98 \times 10^{-5}$ & $-4.95 \times 10^{-5}$ & $-3.66 \times 10^{-5}$ & $-5.11 \times 10^{-5}$ \\
2B & $4.95 \times 10^{-5}$ & $4.93 \times 10^{-5}$ & $3.63 \times 10^{-5}$ & $5.09 \times 10^{-5}$ \\
3F & $-3.93 \times 10^{-4}$ & $-3.92 \times 10^{-4}$ & $-3.88 \times 10^{-4}$ & $-3.92 \times 10^{-4}$ \\
3B & $3.95 \times 10^{-4}$ & $3.94 \times 10^{-4}$ & $3.90 \times 10^{-4}$ & $3.94 \times 10^{-4}$ \\
4F & $-5.82 \times 10^{-4}$ & $-5.82 \times 10^{-4}$ & $-6.27 \times 10^{-4}$ & $-5.81 \times 10^{-4}$ \\
4B & $5.86 \times 10^{-4}$ & $5.86 \times 10^{-4}$ & $6.31 \times 10^{-4}$ & $5.84 \times 10^{-4}$ \\
\hline
\end{tabular}

\section{Numerical studies}

Example 1 A steel shaft having diameter $0.1016 \mathrm{~m}$ and length $1.27 \mathrm{~m}$ supported by two identical isotropic bearings at both the ends is modeled by one BSWI $4_{3}$ rotating Rayleigh-Euler beam element with 33DOFs (Computed by $3 \times 2^{3}+9=33$ ). The material properties of shaft are: Young's modulus $E=2.068 \times 10^{11} \mathrm{~Pa}$, material density $\rho=7833 \mathrm{~kg} / \mathrm{m}$ and Poisson's ratio $\mu=0.3$. Suppose the hysteretic damping $\eta_{H}=0.0002$ and viscous damping $\eta_{V}=0.0002 \mathrm{~s}$, whereas the isotropic bearings having stiffness coefficients $K_{w v}=K_{v w}=0$ and $K_{w w}=K_{v v}=$ $1.7513 \times 10^{7} \mathrm{~N} / \mathrm{m}$.

The present results are found to be in good agreement with those obtained by Zorzi and Nelson [6], Ku [7], Kalita and Kakoty [8] and ÖzgÜven and Özkan [25] as shown in Table 1. The whirl speed maps of this example are presented in Fig. 3(a) and (b), respectively. The letters $F$ and $B$ refer to the forward and backward precessional modes. It has been observed that for the shaft material with hysteretic damping $\eta_{H}=0.0002$, critical speeds for the first three forward modes are found to be $4976 \mathrm{rpm}(\omega=\Omega), 10476 \mathrm{rpm}(\omega=2 \Omega)$ and $21558 \mathrm{rpm}(\omega=3 \Omega)$, respectively, whereas the first three backward natural whirl speeds are $4969 \mathrm{rpm}, 10432 \mathrm{rpm}$ and $21298 \mathrm{rpm}$, respectively. When the viscous damping $\eta_{V}=0.0002 \mathrm{~s}$, the critical speeds for the first and second forward modes are $4989 \mathrm{rpm}$ and $10488 \mathrm{rpm}$, respectively, whereas these are agree with $4960 \mathrm{rpm}$ and $10500 \mathrm{rpm}$ by Ku [7] and $5000 \mathrm{rpm}$ and $10782 \mathrm{rpm}$ by Kalita [8]. The third forward mode is $21270 \mathrm{rpm}$ and the first three backward natural whirl speeds are $4985 \mathrm{rpm}, 10466 \mathrm{rpm}$ and $21184 \mathrm{rpm}$, respectively.

To demonstrate the accuracy of wavelet-based finite element model that considers the effect of bearing damping, a comparison between the present solutions of logarithmic decrements $\delta$ and those which are obtained by using other finite element models, is summarized in Table 2. Suppose the shaft rotating with a specific speed $\Omega=4000 \mathrm{rpm}$ for $\eta_{H}=0.0002$. From Table 2, we can see that the present solutions are in close agreement with the published results, whereas 7 traditional elements with 48 DOFs were used in the published literatures.

Example 2 As a second example, the rotating shaft studied in the first example with the same geometry and material constants is used, but is supported at the two ends by flexible damped bearings. Suppose the rotating speed $\Omega=$ $400 \mathrm{rad} / \mathrm{s}$. The stiffness coefficients of the bearings are $K_{w w}=K_{v v}=1.7513 \times 10^{7} \mathrm{~N} / \mathrm{m}, K_{w v}=K_{v w}=-2.917 \times$ $10^{6} \mathrm{~N} / \mathrm{m}$ and the damping coefficients are $C_{w w}=C_{v v}=1.752 \times 10^{3} \mathrm{Ns} / \mathrm{m}$ and $C_{w v}=C_{v w}=0$. Only one BSWI rotating Rayleigh-Euler shaft element with 33DOFs is used to model the rotating shaft. The natural frequencies $\omega$ and logarithmic decrements $\delta$ of the present element and traditional finite element method with 70 DOFs are 


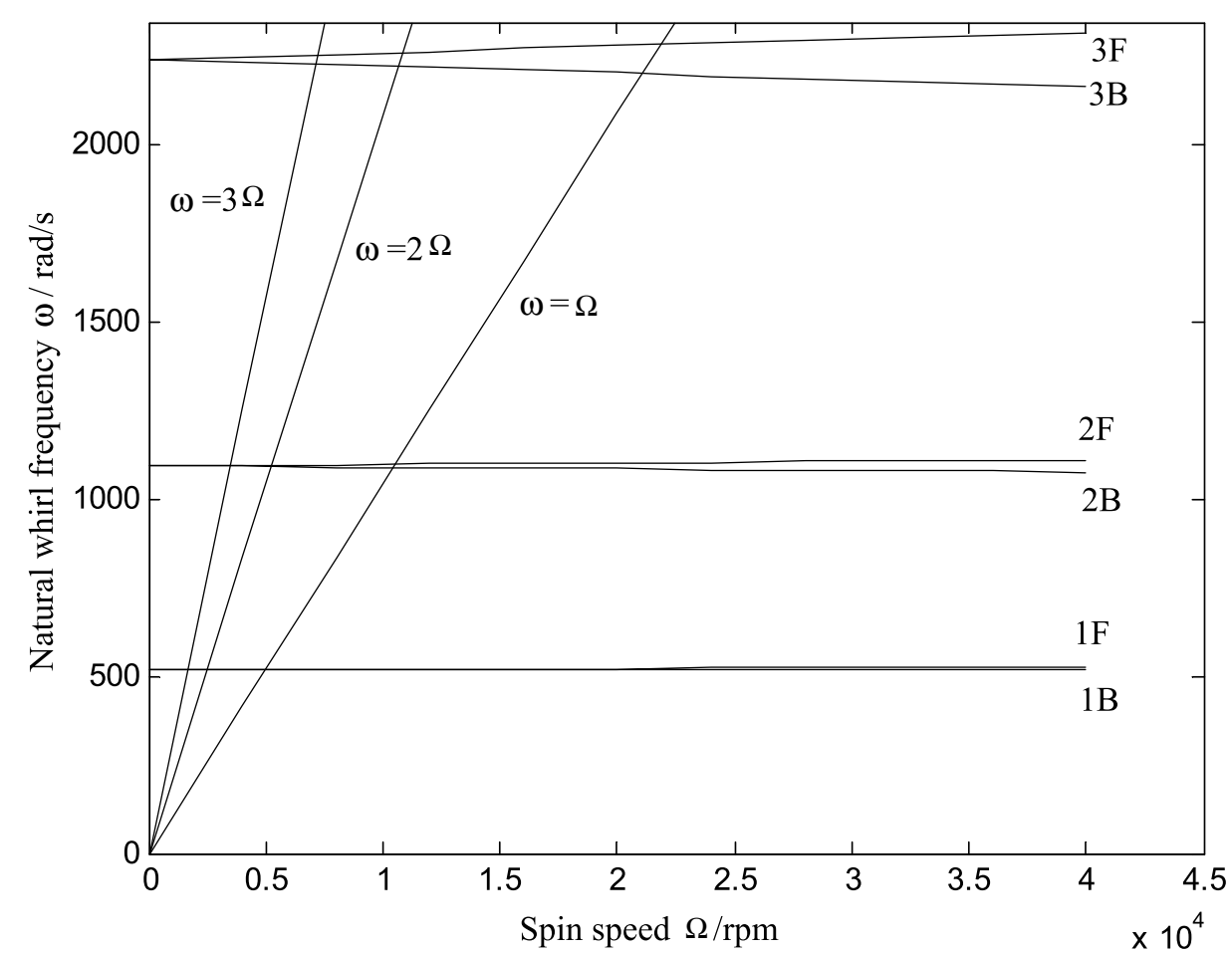

(a)

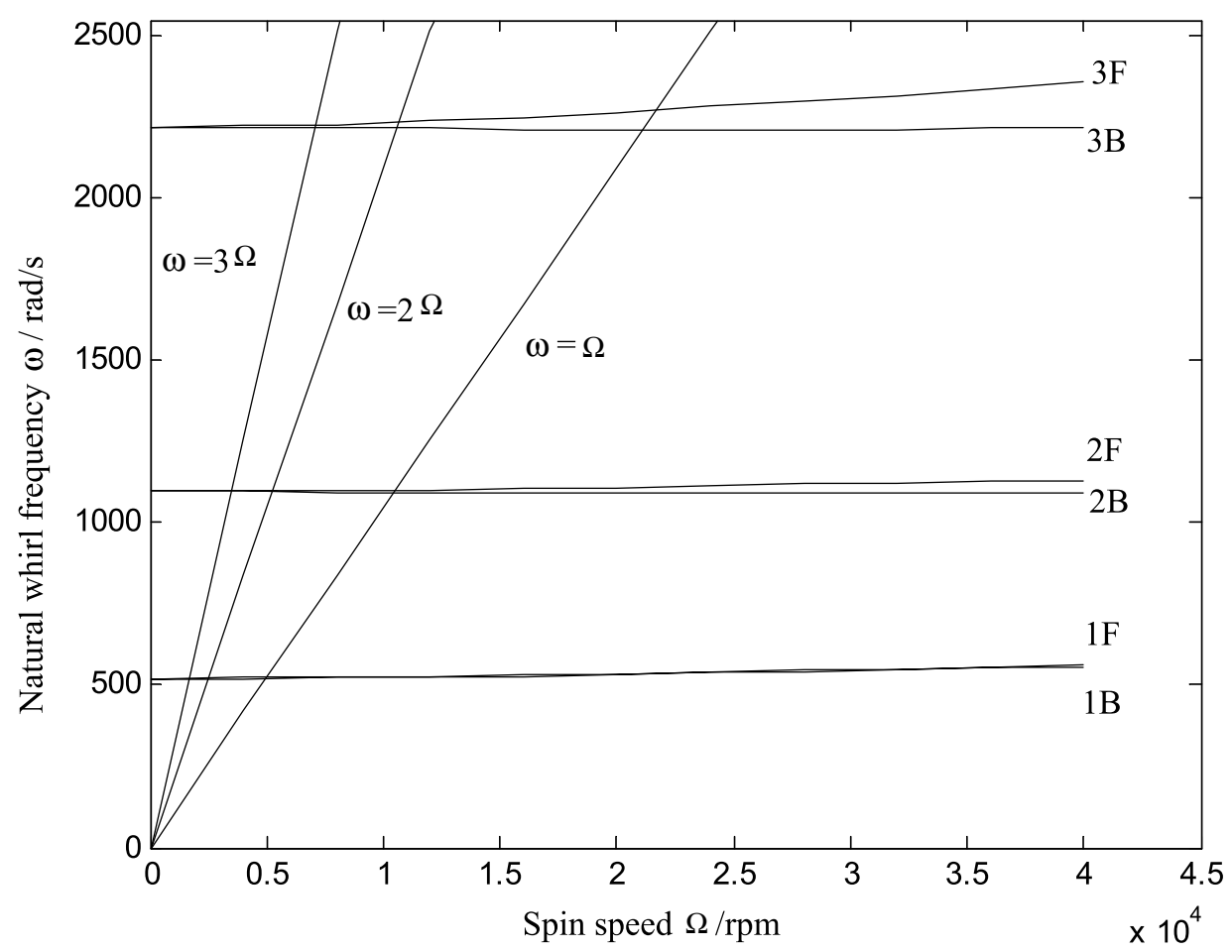

(b)

Fig. 3. Whirl speed maps of a rotor-bearing system supported on undamped isotropic bearing. (a) The shaft material with hysteretic damping $\eta_{H}=0.0002$, and (b) viscous damping $\eta_{V}=0.0002 \mathrm{~s}$. 
Table 3

The comparison of damped frequencies of the rotor system of Example 2

\begin{tabular}{crrrrr}
\hline \multirow{2}{*}{ Mode } & \multicolumn{2}{c}{ Present } & & \multicolumn{2}{c}{ Mohiuddin [26] } \\
\cline { 2 - 3 } \cline { 5 - 6 } & $\omega(\mathrm{rad} / \mathrm{s})$ & \multicolumn{1}{c}{$\delta$} & & $\omega(\mathrm{rad} / \mathrm{s})$ & $\delta$ \\
\hline 1F & 544.76 & 0.0826 & & 544.79 & 0.0826 \\
1B & 491.88 & 0.1208 & & 491.90 & 0.1208 \\
2F & 1173.96 & 0.2877 & & 1174.2 & 0.2879 \\
2B & 1004.87 & 0.3551 & & 1005.0 & 0.3553 \\
3F & 2310.88 & 0.2565 & & 2312.7 & 0.2571 \\
3B & 2170.22 & 0.2710 & & 2171.7 & 0.2715 \\
4F & 5090.73 & 0.1123 & & 5107.4 & 0.1134 \\
4B & 5022.65 & 0.1112 & & 5038.7 & 0.1122 \\
1st torsional mode & 7964.88 & 0.0000003 & & - & - \\
5F & 9552.20 & 0.0549 & & - & - \\
5B & 9489.21 & 0.0546 & & - & - \\
1st axial mode & 12713.07 & 0.0000000007 & & - & - \\
6F & 15413.80 & 0.0318 & & - & - \\
6B & 15330.07 & 0.0317 & & - & - \\
\hline
\end{tabular}

tabulated in Table 3. The comparison shows that the present element produce modal characteristics are in good agree with those of traditional finite element method computed by Mohiuddin, and Khulief [26]. In order to gain the same analytical precision, the present method needs only a half of solving DOFs of traditional finite element method. The first torsional and axial modal frequency are $7964.88 \mathrm{rad} / \mathrm{s}$ and $12713.07 \mathrm{rad} / \mathrm{s}$, respectively, and the corresponding logarithmic decrements $\delta$ is close to the instability threshold.

This numerical example conform the high precision of wavelet-base finite element model. In view of the above, the model can be applied to model rotor-bearing systems, which consider internal damping, bearing stiffness, gyroscopic moments, axial deformation and torsion moment, etc.

\section{Conclusions}

The objective in this paper is to present a novel class of BSWI Rayleigh-Euler rotating beam elements to analyze rotor-bearing system. In this paper, the concepts of the wavelet finite element method and the traditional linear interpolation to formulate BSWI rotating beam element are used. The complex notation and formulation employed have proved to be easily manageable and computationally efficient. Good agreements of numerical examples are obtained between the wavelet-based element and the other published literatures. The proposed wavelet-based elements are suitable to deal with high performance computation for rotor-bearing system. Advantages of the present method over the conventional finite element method are the lower solving DOFs and various wavelet-based elements under different wavelet scales.

\section{Acknowledgements}

Authors are gratefully acknowledging the financial support by the projects of National Natural Science Foundation of China (Nos. 50805028, 50875195), Youth Science Foundation of GuangXi Province of China (No. 0832082), Open Foundation of the State Key Laboratory of Structural Analysis for Industrial Equipment (No. GZ0815), GuangXi Key Laboratory of Manufacturing System \& Advance Manufacturing Technology (No. 0842006_023_Z). We also gratefully thank two anonymous reviewers for their suggestions.

\section{References}

[1] F.F. Ehrich, Handbook of rotordynamics, McGraw-Hill Inc, New York, 1992.

[2] G. Meng, Retrospect and prospect to the research on rotordynamics, Chinese Journal of Vibration Engineering 15(1) (2002), 1-9. (In Chinese) 
[3] W.H. Huang, X.H. Wu, Y.H. Jiao et al., Review of nonlinear rotor dynamics, Chinese Journal of Vibration Engineering 13(4) (2000), 497-509. (In Chinese)

[4] H.D. Nelson, A finite roating shaft element using Timoshenko beam theory, ASME Journal of Mechanical Design 102 (1980), $793-803$.

[5] L.M. Greenhill, W.B. Bickford and H.D. Nelson, A conical beam finite element for rotor dynamic analysis, ASME Journal of Vibration, Acoustics, Stress and Reliability in Design 107 (1985), 421-430.

[6] E.S. Zorzi and H.D. Nelson, Finite element simulation of rotor-bearing systems with internal damping, ASME Journal of Engineering for Power 99 (1977), 71-76.

[7] D.M. Ku, Finite element analysis of whirl speeds for rotor-bearing systems with internal damping, Mechanical Systems and Signal Processing 12 (1998), 599-610.

[8] M. Kalita and S.K. Kakoty, Analysis of whirl speeds for rotor-bearing systems supported on fluid film bearings, Mechanical Systems and Signal Processing 18 (2004), 1369-1380.

[9] S.M. Hashemi, M.J. Richard and G. Dhatt, A new dynamic finite element (DFE) formulation for lateral free vibrations of Euler-Bernoulli spinning beams using trigonometric shape functions, Journal of Sound and Vibration 220(4) (1999), 601-624.

[10] Z.C. Wang, W.L. Cleghorn and S.D. Yu, Free vibration of spinning stepped Timoshenko beams using finite element method, ASME Dynamic Systems and Control Division (Publication) DSC 68 (2000), 17-21.

[11] J.R. Banerjee and H. Su, Development of a dynamic stiffness matrix for free vibration analysis of spinning beams, Computers and Structures 82 (2004), 2189-2197.

[12] J.R. Banerjee and H. Su, Dynamic stiffness formulation and free vibration analysis of a spinning composite beam, Computers and Structures 84 (2006), 1208-1214.

[13] C. Canuto, A. Tabacco and K. Urban, The wavelet element method part I: construction and analysis, Applied and Computational Harmonic Analysis 6 (1999), 1-52.

[14] C. Canuto, A. Tabacco and K. Urban, The wavelet element method part II: realization and additional feature in 2D and 3D, Applied and Computational Harmonic Analysis 8 (2000), 123-165.

[15] J.W. Xiang, Z.J. He and X.F. Chen, The construction of wavelet-based truncated conical shell element using B-spline wavelet on the interval, Acta Mechanica Solida Sinica 19 (2006), 316-326.

[16] J.W. Xiang, X.F. Chen, Z.J. He et al., The construction of 1D wavelet finite elements for structural analysis, Computational Mechanics 40 (2007), 325-339.

[17] J.W. Xiang, X.F. Chen, Z.J. He et al., A new wavelet-based thin plate element using B-spline wavelet on the interval, Computational Mechanics 41 (2008), 243-255.

[18] J.W. Xiang, X.F. Chen, L.F. Yang and Z.J. He, A class of wavelet-based flat shell elements using B-spline wavelet on the interval and its applications, CMES-Computer Modeling in Engineering and Sciences 23 (2008), 1-12.

[19] J.G. Han, W.X. Ren and Y. Huang, A spline wavelet finite element method in structural mechanics, International Journal for Numerical Method in Engineering 66 (2006), 166-190.

[20] J.W. Xiang, X.F. Chen, Z.J. He et al., Identification of crack in a beam based on finite element method of B-spline wavelet on the interval, Journal of Sound and Vibration 296 (2006), 1046-1052

[21] J.W. Xiang, X.F. Chen, Z.J. He et al., Identification of crack in a rotor system based on wavelet finite element method, Finite Elements in Analysis and Design 43 (2007), 1068-1081.

[22] J.C. Goswami, A.K. Chan and C.K. Chui, On solving first-kind integral equations using wavelets on a bounded interval, IEEE Transactions on Antennas and Propagation 43 (1995), 614-622.

[23] C.K. Chui and E. Quak, Wavelets on a bounded interval, Numerical Methods of Approximation Theory 1 (1992), 53-57.

[24] L.M. Greenhill, W.B. Bickford and H.D. Nelson, A conical beam finite element for rotor dynamic analysis, ASME Journal of Vibration, Acoustics, Stress and Reliability in Design 107 (1985), 421-430.

[25] H.N. ÖzgÜven and Z.L. Özkan, Whirl speeds and unbalances response of multi-bearing rotors using finite elements, ASME Journal of Vibration, Stress and Reliability in Design 106 (1984), 72-79.

[26] M.A. Mohiuddin and Y.A. Khulief, Coupled bending trosional vibration of rotors using finite element, Journal of Sound and Vibration 223 (1999), 297-316 

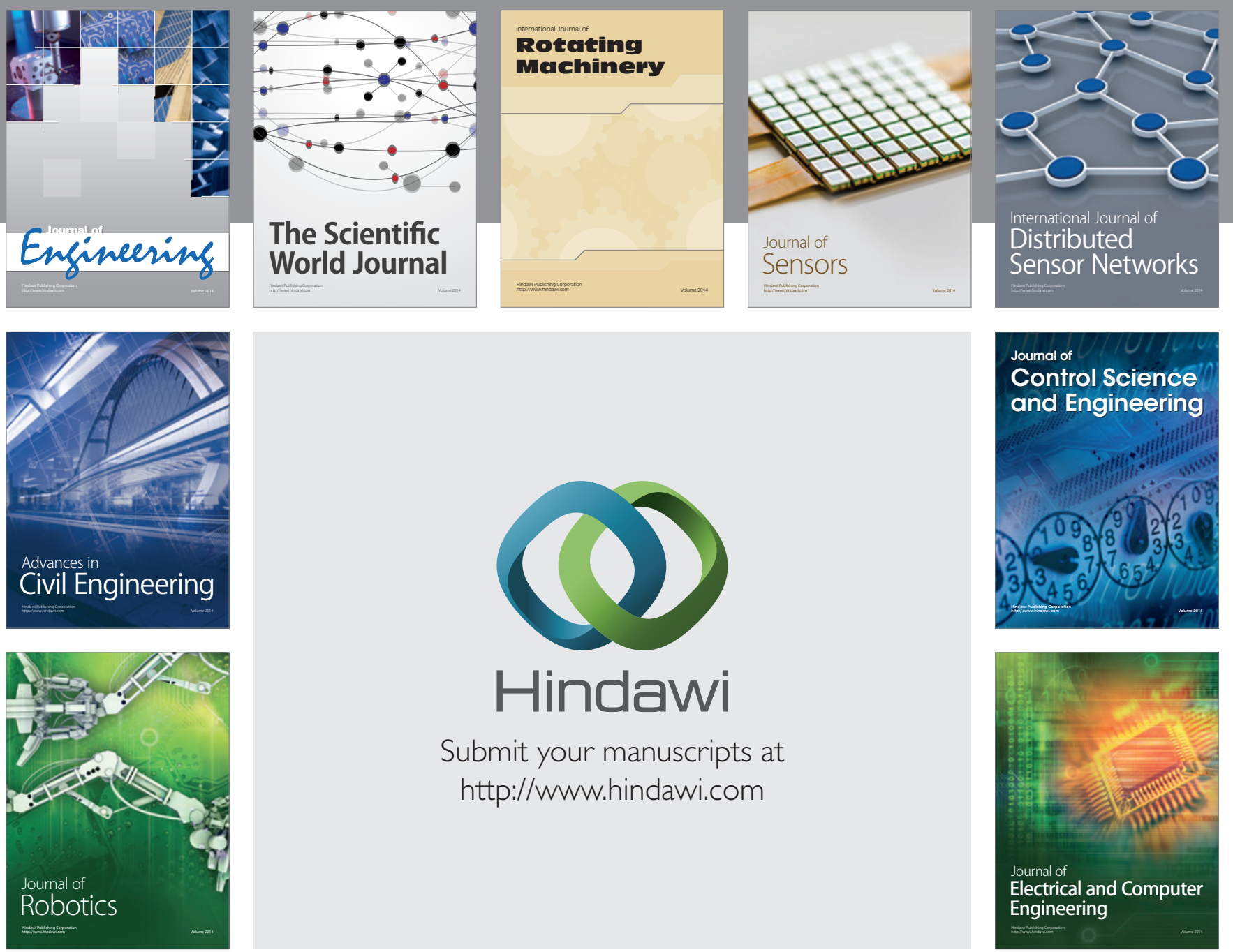

Submit your manuscripts at

http://www.hindawi.com
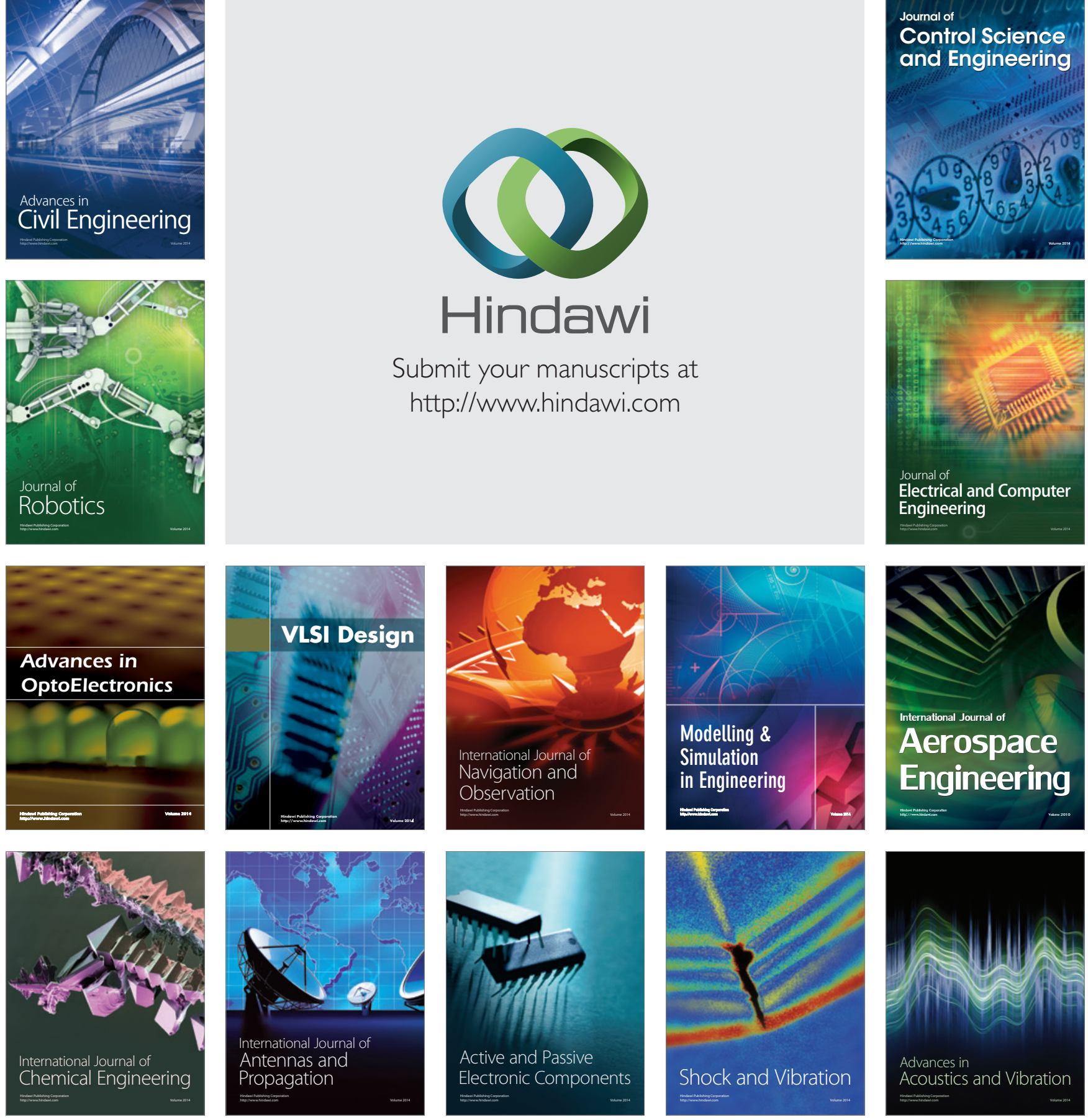